PROCEEDINGS OF THE AMERICAN MATHEMATICAL SOCIETY

Volume 126, Number 5, May 1998, Pages 1549-1555

S 0002-9939(98)04378-0

\title{
ON SOME NEW IDEALS ON THE CANTOR AND BAIRE SPACES
}

\author{
JACEK CICHOŃ AND JAN KRASZEWSKI
}

(Communicated by Andreas R. Blass)

\begin{abstract}
We define and investigate some new ideals of subsets of the Cantor space and the Baire space. We show that combinatorial properties of these ideals can be described by the splitting and reaping cardinal numbers. We show that there exist perfect Luzin sets for these ideals on the Baire space.
\end{abstract}

\section{INTRODUCTION}

For each infinite subset $T$ of the set $\omega$ of all natural numbers let us denote by $\mathcal{K}(T)$ the $\sigma$-ideal of meagre subsets of the space $2^{T}$ with the canonical product topology. By $\mathcal{L}(T)$ we denote the $\sigma$-ideal of Lebesgue measure zero subsets of $2^{T}$ with respect to the canonical product measure.

Notice that if $T$ is a subset of $\omega$ then we can indentify the spaces $2^{T} \times 2^{\omega \backslash T}$ and the Cantor space $2^{\omega}$ using the canonical homeomorphism $\pi_{T}$ defined by $\pi_{T}(x)=$ $(x|T, x|(\omega \backslash T))$. Directly from the definition of meagre sets it follows that if $A \in$ $\mathcal{K}(T)$, then $A \times 2^{\omega \backslash T} \in \mathcal{K}(\omega)$. The same observation is also true for the ideal $\mathcal{L}(\omega)$, but it is evidently false for the $\sigma$-ideal of all countable subsets of the Cantor space. We call this property of the ideals $\mathcal{K}(\omega)$ and $\mathcal{L}(\omega)$ productivity.

There are other natural productive $\sigma$-ideals of subsets of the Cantor space, e.g. the $\sigma$-ideal $\mathcal{K}(\omega) \cap \mathcal{L}(\omega)$. It is interesting that among them there exists the least productive $\sigma$-ideal which contains all points. We call this ideal $S_{2}$. There exists also the least productive ideal of subsets of $2^{\omega}$, and we call it $I_{2}$. These ideals have Borel bases but they do not satisfy the countable chain condition - there exists a family of continuum many pairwise disjoint Borel sets outside the ideal $S_{2}$. The ideal $I_{2}$ is not $\sigma$-additive, and the ideal $S_{2}$ is precisely $\sigma$-additive. The minimum cardinality of bases of these ideals is continuum. The covering number cov of both ideals is equal to the reaping cardinal $\mathfrak{r}$, and the last basic combinatorial invariants (cardinal numbers non) are described in terms of splitting cardinal numbers. These results show that both splitting and reaping cardinals are closely connected with natural mathematical objects on the classical Cantor space.

We also consider the minimal productive $\sigma$-ideal $S_{\omega}$ of subsets of the Baire space

Received by the editors December 18, 1995 and, in revised form, October 16, 1996.

1991 Mathematics Subject Classification. Primary 04A20, 28A05.

Key words and phrases. Set theory, ideals, Borel sets, Cantor space, Baire space, cardinal functions.

Research of the second author supported by a grant 2149/W/IM/96 from the University of Wrocław.

(C) 1998 American Mathematical Society 
$\omega^{\omega}$. We show that there exists an uncountable closed subset $P$ of $\omega^{\omega}$ which is a Luzin set for $S_{\omega}$, i.e. the intersection of the set $P$ with any set from $S_{\omega}$ is countable. This fact completely determines the basic combinatorial invariants of this ideal.

\section{Notation, DEFInitions AND BASIC OBSERVATIONS}

In this paper we use the standard set theoretical notation. For example, $\omega$ denotes the first infinite cardinal number, which we shall identify with the set of all natural numbers. The cardinality of the set of all real numbers is denoted by $\mathfrak{c}$. If $\kappa$ is a cardinal number then $[X]^{\kappa}$ denotes the family of all subsets of $X$ of cardinality $\kappa$ and $[X]^{<\kappa}$ denotes the family of all subsets of $X$ of cardinality strictly less then $\kappa . X^{<\omega}$ denotes the set of all finite sequences of elements of the set $X$. The power set of a set $X$ is denoted by $P(X)$. For $A, B \subseteq \omega$ we put $A \subseteq{ }^{*} B$ if and only if $\operatorname{card}(A \backslash B)<\omega$.

If $X$ is a discrete topological space then we endow $X^{\omega}$ with the standard product topology. In particular, for $X=2$ and $X=\omega$ we get the Cantor space and the Baire space, respectively.

For an ideal $\mathcal{J}$ of subsets of $X$ we consider the following cardinal numbers:

$$
\begin{aligned}
\operatorname{add}(\mathcal{J}) & =\min \{\operatorname{card}(\mathcal{A}): \mathcal{A} \subseteq \mathcal{J} \text { and } \bigcup \mathcal{A} \notin \mathcal{J}\}, \\
\operatorname{cov}(\mathcal{J}) & =\min \{\operatorname{card}(\mathcal{A}): \mathcal{A} \subseteq \mathcal{J} \text { and } \bigcup \mathcal{A}=X\}, \\
\operatorname{non}(\mathcal{J}) & =\min \{\operatorname{card}(B): B \subseteq X \text { and } B \notin \mathcal{J}\}, \\
\operatorname{cof}(\mathcal{J}) & =\min \{\operatorname{card}(\mathcal{A}): \mathcal{A} \subseteq \mathcal{J} \text { and }(\forall A \in \mathcal{J})(\exists B \in \mathcal{A})(A \subseteq B)\} .
\end{aligned}
$$

Note that if $\mathcal{J}$ is a proper ideal and $\bigcup \mathcal{J}=X$, then the following relations hold:

$$
\operatorname{add}(\mathcal{J}) \leq \operatorname{cov}(\mathcal{J}), \operatorname{add}(\mathcal{J}) \leq \operatorname{non}(\mathcal{J}), \operatorname{cov}(\mathcal{J}) \leq \operatorname{cof}(\mathcal{J}), \operatorname{non}(\mathcal{J}) \leq \operatorname{cof}(\mathcal{J}) .
$$

Suppose that $\mathcal{J}$ is an ideal of subsets of $X$ and $\mathcal{A} \subseteq P(X)$. We say that $\mathcal{J}$ has an $\mathcal{A}$-base if for each $A \in \mathcal{J}$ there exists $B \in \mathcal{A} \cap \mathcal{J}$ such that $A \subseteq B$. Hence, in particular, if $X$ is a topological space, then $\mathcal{J}$ has an $F_{\sigma}$-base if each element from $\mathcal{J}$ can be covered by some $F_{\sigma}$ subset of $X$ from $\mathcal{J}$.

Let $\mathcal{K}$ and $\mathcal{L}$ denote the $\sigma$-ideals of meagre subsets and of Lebesgue measure zero subsets of the Cantor space $2^{\omega}$, respectively. The ideal $\mathcal{K}$ has an $F_{\sigma}$-base and the ideal $\mathcal{L}$ has a $G_{\delta}$-base.

From now on let us assume that $X$ has at least two elements. We define

$$
\operatorname{Pif}(X)=\left\{\varphi: \varphi \text { is a function and } \operatorname{dom}(\varphi) \in[\omega]^{\omega} \text { and } \operatorname{rng}(\varphi) \subseteq X\right\} .
$$

If $\varphi \in \operatorname{Pif}(X)$, then we put

$$
\begin{aligned}
& {[\varphi]_{X}=\left\{x \in X^{\omega}: \varphi \subseteq x\right\},} \\
& {[\varphi]_{X}^{*}=\left\{x \in X^{\omega}: \varphi \subseteq^{*} x\right\} .}
\end{aligned}
$$

If we treat $X$ as a discrete topological space, then $[\varphi]_{X}$ is a closed and $[\varphi]_{X}^{*}$ is an $F_{\sigma}$ subset of $X^{\omega}$ for each $\varphi \in \operatorname{Pif}(X)$.

Now we are able to define the ideals we are going to deal with. Let $I_{X}$ and $I_{X}^{*}$ denote the ideals generated by the families $\left\{[\varphi]_{X}: \varphi \in \operatorname{Pif}(X)\right\}$ and $\left\{[\varphi]_{X}^{*}: \varphi \in\right.$ $\operatorname{Pif}(X)\}$, respectively. Then $[X]^{<\omega} \subseteq I_{X} \subseteq I_{X}^{*}$ and $I_{X}^{*}$ is a proper ideal of subsets of $X^{\omega}$. The first ideal has a closed base and the second has an $F_{\sigma}$-base.

Similarly, let $S_{X}$ and $S_{X}^{*}$ denote the $\sigma$-ideals generated by families $\left\{[\varphi]_{X}: \varphi \in\right.$ $\operatorname{Pif}(X)\},\left\{[\varphi]_{X}^{*}: \varphi \in \operatorname{Pif}(X)\right\}$, respectively. It is easy to see that $S_{X}=S_{X}^{*}$. 
Hence we have $I_{X} \subseteq I_{X}^{*} \subseteq S_{X}$. The ideal $S_{X}$ has an $F_{\sigma}$-base and is proper. Directly from the definition of the ideals we can deduce that

$$
\operatorname{cov}\left(I_{X}\right)=\operatorname{cov}\left(I_{X}^{*}\right)=\operatorname{cov}\left(S_{X}\right) .
$$

If $j: \omega \rightarrow \omega$ is an injection and $A \subseteq 2^{\omega}$, then we define

$$
j * A=\left\{x \in 2^{\omega}: x \circ j \in A\right\} .
$$

We say that an ideal $\mathcal{J}$ of subsets of $2^{\omega}$ is productive if $j * A \in \mathcal{J}$ for each $A \in \mathcal{J}$ and any injection $j: \omega \rightarrow \omega$. As we have mentioned in the introduction, the ideals $\mathcal{K}$ and $\mathcal{L}$ are productive.

Let $\varphi \in \operatorname{Pif}(2)$ and let $j: \omega \rightarrow \operatorname{dom}(\varphi)$ be any bijection. Then $j *\{\varphi \circ j\}=[\varphi]_{2}$. Conversely, if $j: \omega \rightarrow \omega$ is an injection and $x \in 2^{\omega}$, then $x \circ j^{-1} \in \operatorname{Pif}(2)$ and

$$
j *\{x\}=\left[x \circ j^{-1}\right]_{2} .
$$

These observations imply that $I_{2}$ and $S_{2}$ are the least productive ideal and $\sigma$-ideal of subsets of $2^{\omega}$ (containing all points), respectively. Since $\mathcal{K}$ and $\mathcal{L}$ are productive, we see that $S_{2} \subseteq \mathcal{K} \cap \mathcal{L}$.

Let us recall that $\sigma$-ideals of subsets of a Polish space with Borel bases have plenty of interesting properties (see e.g. [2]). The next result shows the main difference between the ideal $S_{2}$ and the ideals $\mathcal{K}$ and $\mathcal{L}$.

Theorem 1.1. There exists a family $\mathcal{F}$ of pairwise disjoint Borel subsets of $2^{\omega}$ such that $\operatorname{card}(\mathcal{F})=\mathfrak{c}$ and none of elements of $\mathcal{F}$ belongs to $S_{2}$.

Proof. For each real number $\alpha \in[0,1]$ we put

$$
A_{\alpha}=\left\{x \in 2^{\omega}: \lim _{n \rightarrow \infty} \frac{1}{n} \sum_{i=1}^{n} x(i)=\alpha\right\} .
$$

It is easy to check that $\left\{A_{\alpha}: \alpha \in[0,1]\right\}$ is the required family.

Let $A, S$ be two infinite subsets of $\omega$. We say that $S$ splits $A$ if $\operatorname{card}(A \cap S)=$ $\operatorname{card}(A \backslash S)=\omega$. Let us recall the following three cardinal numbers (see e.g. [3]):

$$
\begin{aligned}
\mathfrak{s} & =\min \left\{\operatorname{card}(\mathcal{S}): \mathcal{S} \subseteq[\omega]^{\omega} \text { and }\left(\forall A \in[\omega]^{\omega}\right)(\exists S \in \mathcal{S})(S \text { splits } A)\right\}, \\
\aleph_{0-\mathfrak{s}} & =\min \left\{\operatorname{card}(\mathcal{S}): \mathcal{S} \subseteq[\omega]^{\omega} \text { and }\left(\forall \mathcal{A} \in\left[[\omega]^{\omega}\right]^{\omega}\right)(\exists S \in \mathcal{S})(\forall A \in \mathcal{A})(S \text { splits } A)\right\}, \\
\mathfrak{r} & =\min \left\{\operatorname{card}(\mathcal{R}): \mathcal{R} \subseteq[\omega]^{\omega} \text { and }\left(\forall S \in[\omega]^{\omega}\right)(\exists R \in \mathcal{R})(S \text { does not split } R)\right\} .
\end{aligned}
$$

The cardinal numbers $\mathfrak{s}$ and $\mathfrak{r}$ are called splitting and reaping, respectively. It is easy to check that $\omega_{1} \leq \mathfrak{s} \leq \aleph_{0} \mathfrak{s} \leq \mathfrak{c}$ and $\omega_{1} \leq \mathfrak{r} \leq \mathfrak{c}$. It is an open problem if $\mathfrak{s}=\aleph_{0}-\mathfrak{s}$ can be proved in ZFC. An easy reformulation of the definition of the reaping number $\mathfrak{r}$ gives us the following description:

$$
\mathfrak{r}=\min \left\{\operatorname{card}(\mathcal{R}): \mathcal{R} \subseteq[\omega]^{\omega} \text { and }\left(\forall S \in[\omega]^{\omega}\right)(\exists R \in \mathcal{R})(R \subseteq S \vee R \subseteq \omega \backslash S)\right\} .
$$

Let us introduce an auxiliary cardinal number. Namely, we define

$$
\begin{aligned}
\operatorname{fin}-\mathfrak{s}=\min \{\operatorname{card}(\mathcal{S}) & : \mathcal{S} \subseteq[\omega]^{\omega} \\
& \text { and } \left.\left(\forall \mathcal{A} \in\left[[\omega]^{\omega}\right]^{<\omega}\right)(\exists S \in \mathcal{S})(\forall A \in \mathcal{A})(S \text { splits } A)\right\} .
\end{aligned}
$$

Lemma 1.2. $\mathfrak{s}=$ fin- $\mathfrak{s}$. 
Proof. It is clear that $\mathfrak{s} \leq$ fin-s. Suppose now that $\mathcal{S} \subseteq[\omega]^{\omega}$ is such that $\operatorname{card}(\mathcal{S})=$ $\mathfrak{s}$ and every infinite subset $A \subseteq \omega$ is split by some element of $\mathcal{S}$. We may assume that $\mathcal{S}$ is a field of subsets of $\omega$ containing all finite sets. One can show by an easy induction on $n \in \omega$ that

$$
\left.\left(\forall \mathcal{A} \in\left[[\omega]^{\omega}\right]^{n}\right)(\exists S \in \mathcal{S})(\forall A \in \mathcal{A})(S \text { splits } A)\right\} .
$$

\section{BASIC PROPERTIES}

Assume that $X \subseteq Y, \operatorname{card}(X) \geq 2$. Then it is easy to see that

$$
I_{X}=I_{Y} \cap P\left(X^{\omega}\right), \quad I_{X}^{*}=I_{Y}^{*} \cap P\left(X^{\omega}\right) \quad \text { and } \quad S_{X}=S_{Y} \cap P\left(X^{\omega}\right) .
$$

Let us recall a well-known fact.

Lemma 2.1. Suppose that $\mathcal{I}$ is an ideal of subsets of $X, \mathcal{J}$ is an ideal of subsets of $Y, X \subseteq Y$ and $\mathcal{I}=\mathcal{J} \cap P(X)$. Then $\operatorname{cov}(\mathcal{I}) \leq \operatorname{cov}(\mathcal{J})$ and $\operatorname{non}(\mathcal{J}) \leq \operatorname{non}(\mathcal{I})$.

For each set $X$ we have

Lemma 2.2. $\operatorname{add}\left(I_{X}\right)=\operatorname{non}\left(I_{X}\right)=\omega$.

Proof. Lemma 2.1 implies that we have to prove non $\left(I_{2}\right)=\omega$ only. Let

$$
A=\left\{x \in 2^{\omega}:\left(\forall^{\infty} n \in \omega\right) x(n)=0\right\} .
$$

Then $\operatorname{card}(A)=\omega$, and it is easy to check that $A \notin I_{2}$.

We call a family $\mathcal{F} \subseteq \operatorname{Pif}(X)$ normal if for each two different $\varphi_{1}, \varphi_{2} \in \mathcal{F}$ we have $\operatorname{dom}\left(\varphi_{1}\right) \cap \operatorname{dom}\left(\varphi_{2}\right)=\emptyset$. Notice that if $\left\{\varphi_{i}: i \in I\right\} \subseteq \operatorname{Pif}(X)$ and $\operatorname{card}(X) \leq \omega$, then there exists a normal family $\left\{\psi_{i}: i \in I\right\}$ such that $\psi_{i} \subseteq \varphi_{i}$ for each $i \in I$. Notice also that if $\varphi, \psi \in \operatorname{Pif}(X)$ and $\psi \subseteq \varphi$, then $[\varphi]_{X} \subseteq[\psi]_{X}$ and $[\varphi]_{X}^{*} \subseteq[\psi]_{X}^{*}$. Therefore for every $A \in X^{\omega}$ we have

1) $A \in I_{X} \Longleftrightarrow A \subseteq \bigcup_{\varphi \in \mathcal{F}}[\varphi]_{X}$ for some finite normal family $\mathcal{F} \subseteq \operatorname{Pif}(X)$,

2) $A \in I_{X}^{*} \Longleftrightarrow A \subseteq \bigcup_{\varphi \in \mathcal{F}}[\varphi]_{X}^{*}$ for some finite normal family $\mathcal{F} \subseteq \operatorname{Pif}(X)$,

3) $A \in S_{X} \Longleftrightarrow A \subseteq \bigcup_{\varphi \in \mathcal{F}}[\varphi]_{X}$ for some countable normal family $\mathcal{F} \subseteq \operatorname{Pif}(X)$.

Lemma 2.3. Suppose that $\left\{\varphi_{i}: i \in I\right\}$ is a normal family of functions from $\operatorname{Pif}(X), \varphi \in \operatorname{Pif}(X)$ and

$$
[\varphi]_{X} \subseteq \bigcup_{i \in I}\left[\varphi_{i}\right]_{X} \quad\left([\varphi]_{X}^{*} \subseteq \bigcup_{i \in I}\left[\varphi_{i}\right]_{X}^{*}\right)
$$

Then $[\varphi]_{X} \subseteq\left[\varphi_{i}\right]_{X} \quad\left([\varphi]_{X}^{*} \subseteq\left[\varphi_{i}\right]_{X}^{*}\right)$ for some $i \in I$.

Proof. We shall prove only the second case of the lemma. Suppose that $[\varphi]_{X}^{*} \nsubseteq$ $\left[\varphi_{i}\right]_{X}^{*}$ for each $i \in I$, i.e $\varphi_{i} \mathbb{}^{*} \varphi$ for each $i \in I$. Hence, we may find a family $\left\{K_{i}: i \in I\right\}$ of infinite subsets of $\omega$ such that

$$
\begin{aligned}
& \text { 1) } K_{i} \subseteq \operatorname{dom}\left(\varphi_{i}\right), \\
& \text { 2) }\left(\forall k \in K_{i}\right)\left(k \notin \operatorname{dom}(\varphi) \vee \varphi_{i}(k) \neq \varphi(k)\right)
\end{aligned}
$$

for each $i \in I$. Notice that elements from the family $\left\{K_{i}: i \in I\right\}$ are pairwise disjoint. We consider an arbitrary function $f \in X^{\omega}$ such that $\varphi \subseteq f$ and $f(k) \neq$ 
$\varphi_{i}(k)$ for each $k \in K_{i} \backslash \operatorname{dom}(\varphi)$ and $i \in I$. One can show with ease that $f \in$ $[\varphi]_{X}^{*} \backslash \bigcup_{i \in I}\left[\varphi_{i}\right]_{X}^{*}$, which leads to a contradiction.

The proof of the first case of the lemma is similar; infinite sets $K_{i}$ should be replaced by singletons.

Let $\mathcal{I}$ be an arbitrary ideal and let $\kappa \leq \lambda$ be two infinite cardinals. A family $\mathcal{A} \subseteq$ $\mathcal{I}$ is called a $(\kappa, \lambda)$-family for $\mathcal{I}$ if $\operatorname{card}(\mathcal{A})=\lambda$ and $\operatorname{card}(\{A \in \mathcal{A}: A \subseteq S\})<\kappa$ for each $S \in \mathcal{I}$.

Lemma 2.4. Let card $(X) \geq 2$. Then there exists a family $\mathcal{A} \subseteq I_{X}$ which is an $(\omega, \mathfrak{c})$-family for $I_{X}^{*}$ and an $\left(\omega_{1}, \mathfrak{c}\right)$-family for $S_{X}$.

Proof. We may assume that $\{0,1\} \subseteq X$. Let us fix a family $\mathcal{F} \subseteq[\omega]^{\omega}$ of cardinality $\mathfrak{c}$ such that $\operatorname{card}(A \cap B)<\omega$ for any two different $A, B \in \mathcal{F}$. Let $\mathcal{A}=\left\{[A \times\{1\}]_{X}\right.$ : $A \in \mathcal{F}\}$. It is clear that $\mathcal{A} \subseteq I_{X}$. Suppose now that $S \in I_{X}^{*}$. Then for some $k \in \omega$ and a normal family $\left\{\varphi_{1}, \ldots, \varphi_{k}\right\} \subseteq \operatorname{Pif}(X)$ we have $S \subseteq\left[\varphi_{1}\right]_{X}^{*} \cup \cdots \cup\left[\varphi_{k}\right]_{X}^{*}$. Let $\mathcal{Y}=\left\{A \in \mathcal{F}:[A \times\{1\}]_{X}^{*} \subseteq\left[\varphi_{1}\right]_{X}^{*} \cup \cdots \cup\left[\varphi_{k}\right]_{X}^{*}\right\}$. We claim that $\operatorname{card}(\mathcal{Y}) \leq k$. Suppose otherwise. Notice that Lemma 2.3 implies

$$
\mathcal{Y}=\left\{A \in \mathcal{F}:(\exists i \in\{1, \ldots, k\})\left([A \times\{1\}]_{X}^{*} \subseteq\left[\varphi_{i}\right]_{X}^{*}\right)\right\} .
$$

Therefore, there are two different $A, B \in \mathcal{F}$ and $i \in\{1, \ldots, k\}$ such that

$$
[A \times\{1\}]_{X}^{*} \subseteq\left[\varphi_{i}\right]_{X}^{*}, \quad[B \times\{1\}]_{X}^{*} \subseteq\left[\varphi_{i}\right]_{X}^{*} .
$$

But then $\varphi_{i} \subseteq^{*} A \times\{1\}$ and $\varphi_{i} \subseteq^{*} B \times\{1\}$; hence $\operatorname{card}(A \cap B)=\omega$. So we have obtained a contradiction. The proof of the other part of the lemma is similar.

It is easy to check (see e.g. [1]) that if there exists a $(\kappa, \lambda)$-family for an ideal $\mathcal{I}$ and $\kappa<\lambda$ (or $\kappa=\lambda$ and $\kappa$ is regular), then $\operatorname{add}(\mathcal{I}) \leq \kappa$ and $\operatorname{cof}(\mathcal{I}) \geq \lambda$. Hence we obtain the following result.

Theorem 2.5. Let $\operatorname{card}(X) \geq 2$. Then

$$
\operatorname{add}\left(I_{X}^{*}\right)=\omega, \operatorname{add}\left(S_{X}\right)=\omega_{1} .
$$

If moreover $\operatorname{card}(X) \leq \omega$, then

$$
\operatorname{cof}\left(I_{X}\right)=\operatorname{cof}\left(I_{X}^{*}\right)=\operatorname{cof}\left(S_{X}\right)=\mathfrak{c} .
$$

\section{IdEALS ON THE BAIRE SPACE}

In this part we shall discuss the properties of ideals $I_{\omega}, I_{\omega}^{*}$ and $S_{\omega}$. Hence we shall now work on the classical Baire space of infinite sequences of natural numbers. Let us recall that if $\mathcal{I}$ is an ideal of subsets of $X$, then a set $L \subseteq X$ is called a Luzin set for $\mathcal{I}$ if $\operatorname{card}(L)=\operatorname{card}(X)$ and $\operatorname{card}(A \cap L) \leq \omega$ for each $A \in \mathcal{I}$. Notice that if there exists a Luzin set for an ideal $\mathcal{I}$, then $\operatorname{non}(\mathcal{I}) \leq \omega_{1}$ and $\operatorname{cov}(\mathcal{I})=\operatorname{card}(X)$.

A subset of a topological space is perfect if it is closed and contains no isolated points.

Theorem 3.1. There exists a perfect Luzin set for the ideal $S_{\omega}$.

Proof. Fix a bijection $b: 2^{<\omega} \rightarrow \omega$. To each $f \in 2^{\omega}$ associate $\tilde{f}: \omega \rightarrow \omega$ defined by $\tilde{f}(n)=b(f(0), \ldots, f(n-1))$. Then $\left\{\tilde{f}: f \in 2^{\omega}\right\}$ is a perfect set, and it is Luzin for $S_{\omega}$ because no two of its members agree infinitely often. 
Putting together Lemma 2.2, Theorem 2.5, Theorem 3.1 and the observations at the beginning of this part, we are able to describe the cardinal coefficients add, non, cov, cof of the ideals $I_{\omega}, I_{\omega}^{*}, S_{\omega}$.

Theorem 3.2. 1) $\operatorname{add}\left(I_{\omega}\right)=\operatorname{add}\left(I_{\omega}^{*}\right)=\omega<\operatorname{add}\left(S_{\omega}\right)=\omega_{1}$;

2) $\operatorname{non}\left(I_{\omega}\right)=\operatorname{non}\left(I_{\omega}^{*}\right)=\omega<\operatorname{non}\left(S_{\omega}\right)=\omega_{1}$;

3) $\operatorname{cov}\left(I_{\omega}\right)=\operatorname{cov}\left(I_{\omega}^{*}\right)=\operatorname{cov}\left(S_{\omega}\right)=\mathfrak{c}$;

4) $\operatorname{cof}\left(I_{\omega}\right)=\operatorname{cof}\left(I_{\omega}^{*}\right)=\operatorname{cof}\left(S_{\omega}\right)=\mathfrak{c}$.

\section{Ideals on the CANTOR SPACES}

In this section we shall discuss the ideals $I_{n}, I_{n}^{*}$ and $S_{n}$ for natural numbers $n \geq 2$. Let us recall that we identify a number $n$ with the set $\{0, \ldots, n-1\}$. Hence, for example, the ideals $I_{2}, I_{2}^{*}$ and $S_{2}$ are ideals of subsets of the classical Cantor space.

Lemma 4.1. Let $2 \leq n \leq \omega$. Then $\operatorname{cov}\left(I_{n}\right)=\operatorname{cov}\left(I_{n}^{*}\right)=\operatorname{cov}\left(S_{n}\right)=\mathfrak{r}$.

Proof. As we have observed in the introduction, the cardinal numbers $\operatorname{cov}\left(I_{n}\right)$, $\operatorname{cov}\left(I_{n}^{*}\right)$ and $\operatorname{cov}\left(S_{n}\right)$ are equal. Hence we shall prove that $\operatorname{cov}\left(S_{n}\right)=\mathfrak{r}$.

Suppose first that $\mathcal{F} \subseteq \operatorname{Pif}(2)$ is a family such that

$$
\bigcup_{\varphi \in \mathcal{F}}[\varphi]=2^{\omega} \text {. }
$$

Let $\mathcal{R}=\left\{\varphi^{-1}[\{i\}]: i \in\{0,1\}\right.$ and $\left.\varphi \in \mathcal{F}\right\} \cap[\omega]^{\omega}$. Then

$$
\left(\forall A \in[\omega]^{\omega}\right)(\exists R \in \mathcal{R})\left(R \subseteq A \vee R \subseteq A^{c}\right),
$$

so $\operatorname{cov}\left(S_{2}\right) \geq \mathfrak{r}$. We get from Lemma 2.1 that $\operatorname{cov}\left(S_{n}\right) \geq \mathfrak{r}$ for each $n \geq 2$.

Now we fix a family $\mathcal{R} \subseteq[\omega]^{\omega}$ of cardinality $\mathfrak{r}$ such that for each $A \in[\omega]^{\omega}$ there exists $R \in \mathcal{R}$ which is contained either in $A$ or in its complement. We can inscribe an isomorphic copy of the whole family $\mathcal{R}$ into each of its elements and repeat this process countably many times. Then we obtain a family $\mathcal{R}^{*} \subseteq[\omega]^{\omega}$ of cardinality $\mathfrak{r}$ such that for each partition of $\omega$ into finitely many pieces there exists $R \in \mathcal{R}^{*}$ which is contained in some element of the partition. Let

$$
\mathcal{F}=\left\{[R \times\{i\}]: i \in\{1, \ldots, n\} \text { and } R \in \mathcal{R}^{*}\right\} .
$$

Then $\mathcal{F} \subseteq S_{n}$ and $\bigcup \mathcal{F}=n^{\omega}$. Hence $\operatorname{cov}\left(S_{n}\right) \leq \mathfrak{r}$.

Lemma 4.2. Let $2 \leq n \leq \omega$. Then $\operatorname{non}\left(I_{n}^{*}\right)=\mathfrak{s}$ and $\operatorname{non}\left(S_{n}\right)=\aleph_{0}-\mathfrak{s}$.

Proof. Suppose that $T \subseteq n^{\omega}, \operatorname{card}(T)=\operatorname{non}\left(I_{n}^{*}\right)$ and $T \notin I_{n}^{*}$. Let

$$
\mathcal{S}=\left\{x^{-1}[\{i\}]: i \in\{1, \ldots, n\} \text { and } x \in T\right\} \cap[\omega]^{\omega} .
$$

We claim that for each $A \in[\omega]^{\omega}$ there exists $S \in \mathcal{S}$ such that $\operatorname{card}(A \cap S)=$ $\operatorname{card}(A \backslash S)=\omega$, and so $\mathfrak{s} \leq \operatorname{card}(\mathcal{S})=\operatorname{non}\left(I_{n}^{*}\right)$. Indeed, let $A \in[\omega]^{\omega}$. Then the set $A^{*}=\bigcup\left\{[A \times\{i\}]^{*}: i \in\{1, \ldots, n\}\right\}$ belongs to the ideal $I_{n}^{*}$, so for some $x \in T$ we have $x \notin A^{*}$. We consider $i<\omega$ such that $\operatorname{card}\left(A \cap x^{-1}[\{i\}]\right)=\omega$. Note that the relation $\operatorname{card}\left(A \backslash x^{-1}[\{i\}]\right)<\omega$ is impossible, since $x \notin A^{*}$. Hence $x^{-1}[\{i\}]$ splits the set $A$. Therefore the inequality $\mathfrak{s} \leq \operatorname{non}\left(I_{n}^{*}\right)$ holds.

We shall prove now that $\operatorname{non}\left(I_{2}^{*}\right) \leq$ fin-s. Suppose that $\mathcal{S} \subseteq[\omega]^{\omega}$ is such a family that $\operatorname{card}(\mathcal{S})=$ fin-s and for each $\mathcal{A} \in\left[[\omega]^{\omega}\right]^{<\omega}$ there exists $S \in \mathcal{S}$ which splits each 
element of $\mathcal{A}$. Then one can check that the set

$$
Y=\{(S \times\{1\}) \cup((\omega \backslash S) \times\{0\}): S \in \mathcal{S}\} \subseteq n^{\omega}
$$

does not belong to $I_{2}^{*}$. Hence non $\left(I_{n}^{*}\right) \leq \operatorname{non}\left(I_{2}^{*}\right) \leq$ fin- $\leq \mathfrak{s}$.

Similarly, $\operatorname{non}\left(S_{2}\right)=\aleph_{0}-\mathfrak{s}$. According to Lemma 2.1, we have

$$
\operatorname{non}\left(S_{2}\right) \geq \operatorname{non}\left(S_{3}\right) \geq \ldots,
$$

and hence it is sufficient to prove that $\operatorname{non}\left(S_{n}\right) \leq \operatorname{non}\left(S_{n+1}\right)$ for each natural $n$. This is easily established by a standard technique from partition calculus, temporarily identifying two elements of $n+1$ to obtain a nearly homogeneous set and then unidentifying those two elements to get homogeneity.

Our last theorem summarizes the properties of ideals $I_{n}, I_{n}^{*}$ and $S_{n}$ for finite $n$ proved in this paper.

Theorem 4.3. Let $2 \leq n \leq \omega$. Then

1) $\operatorname{add}\left(I_{n}\right)=\operatorname{add}\left(I_{n}^{*}\right)=\omega<\operatorname{add}\left(S_{n}\right)=\omega_{1}$

2) $\operatorname{non}\left(I_{n}\right)=\omega<\operatorname{non}\left(I_{n}^{*}\right)=\mathfrak{s} \leq \operatorname{non}\left(S_{n}\right)=\aleph_{0}-\mathfrak{s}$;

3) $\operatorname{cov}\left(I_{n}\right)=\operatorname{cov}\left(I_{n}^{*}\right)=\operatorname{cov}\left(S_{n}\right)=\mathfrak{r}$;

4) $\operatorname{cof}\left(I_{n}\right)=\operatorname{cof}\left(I_{n}^{*}\right)=\operatorname{cof}\left(S_{n}\right)=\mathfrak{c}$.

\section{ACKNOWLEDGEMENTS}

The authors are grateful to Professor Andreas Blass for useful comments on the original version of the paper.

\section{REFERENCES}

1. Cichoń J., On two-cardinal properties of ideals, Trans. Amer. Math. Soc. 314 (1989), 693-708. MR 91a:04001

2. Cichoń J., Kharazishvili A.B., On ideals with projective bases, 1995 (to appear).

3. van Douwen E.K., The integers and topology in Handbook of Set Theoretical Topology, K. Kunen and J. Vaughan, eds., North-Holland, Amsterdam (1984), 111-167. MR 87f:54008

Department of Mathematics, University of Wroceaw, Pl. Grunwaldzki 2/4, 50-156 Wroceaw, Poland

E-mail address: cichon@math.uni.wroc.pl

E-mail address: kraszew@math.uni.wroc.pl 